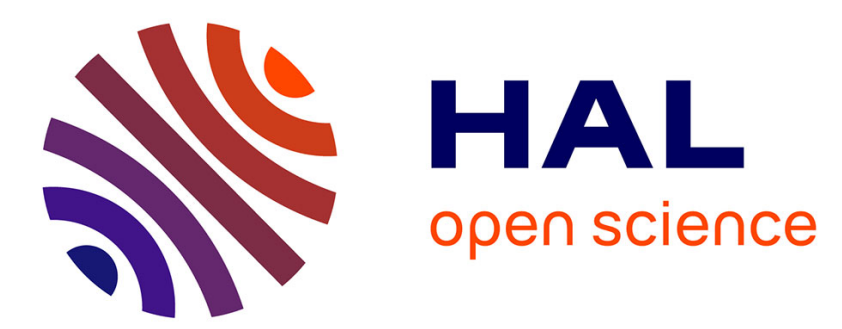

\title{
L'archéologie à Rome après 1870. Une lecture politique et spatiale
}

Denis Bocquet

\section{To cite this version:}

Denis Bocquet. L'archéologie à Rome après 1870. Une lecture politique et spatiale. Mélanges de l'École française de Rome - Italie et Méditerranée, 2001, 113 (2), pp.759-773. halshs-00129556

\section{HAL Id: halshs-00129556 \\ https://shs.hal.science/halshs-00129556}

Submitted on 4 Apr 2007

HAL is a multi-disciplinary open access archive for the deposit and dissemination of scientific research documents, whether they are published or not. The documents may come from teaching and research institutions in France or abroad, or from public or private research centers.
L'archive ouverte pluridisciplinaire HAL, est destinée au dépôt et à la diffusion de documents scientifiques de niveau recherche, publiés ou non, émanant des établissements d'enseignement et de recherche français ou étrangers, des laboratoires publics ou privés. 


\section{L'archéologie à Rome après 1870 : une lecture politique et spatiale}

\section{Denis Bocquet}

Article publié dans les Mélanges de l'Ecole française de Rome (Italie et Méditerranée) (MEFRIM), 2001, vol. 113, p. 759-773.

Pour toute citation, merci de se référer à la version publiée.

Lorsqu'en 1870 Rome est intégrée au Royaume d'Italie, et en devient, en 1871, la capitale, les domaines de l'archéologie et de la préservation du patrimoine antique sont déjà, depuis plusieurs décennies, l'objet d'une attention soutenue et institutionnalisée. Sous le gouvernement pontifical, une Commission des Beaux Arts et des Antiquités avait pour rôle de définir les grandes orientations en la matière, et dans un paysage urbain qui avait déjà commencé d'évoluer, le périmètre qui allait devenir la zone archéologique centrale avait, pour une grande part, déjà été délimité.

La zone comprise entre le Forum, le Palatin, le Colisée et les Thermes de Caracalla était depuis de nombreuses années livrée aux archéologues. Après les campagnes des années 1809-1814, durant les années d'occupation française de la ville, le gouvernement pontifical du Cardinal Consalvi avait pris des dispositions destinées à poursuivre les travaux de dégagement. Malgré de sérieux conflits avec des archéologues comme Fea, inquiets face à la tendance du Secrétaire d'Etat à considérer les Antiquités comme une marchandise sujette au commerce et à la taxation, on peut dire que le périmètre archéologique central fut préservé $^{1}$ En 1820, un édit du 
Camerlengo Pacca, remplaçant le précédent texte de 1802, soumet le secteur à une réglementation stricte ${ }^{2}$. Une Commission est chargée de veiller sur les Antiquités de la ville. En 1827, Léon XII approuve le complet dégagement du Forum jusqu'au niveau du sol antique ${ }^{3}$. Entre les années 1830 et la prise de la ville par l'armée royale italienne, les travaux avancent à un rythme raisonnable, et confirment la place de la zone archéologique dans le paysage urbain de la ville du XIX ${ }^{\mathrm{e}}$ siècle. Les premiers frémissements du marché immobilier et du secteur de la construction, dès avant 1870 , ne menacent pas cet espace de l'archéologie, qui apparaît d'ores et déjà à l'abri, sinon tout à fait de l'avidité des marchands d'Art, au moins de la spéculation foncière.

Avant 1870 , on repère déjà plusieurs types de conflits entre institutions, qui annoncent ceux que l'on va décrire pour les premières décennies de Rome capitale du Royaume d'Italie. L'archéologie a en effet été peu à peu définie, dans la pratique institutionnelle romaine, comme domaine de compétence étatique. Mais les institutions municipales n'avaient en rien renoncé à leurs attributions, fondées sur de solides traditions et bases juridiques. On trouve en effet dès le $\mathrm{XIV}^{\mathrm{e}}$ siècle parmi les compétences de la magistrature capitoline la responsabilité des Antiquités. Dans les statuts de la ville de 1363 apparait, parmi les charges du Senatore, celle de protéger les monuments anciens (De antiquis edificiis non diruendis $)^{4} . \mathrm{Au} \mathrm{XV}^{\mathrm{e}}$ siècle, Pie II semble avoir confirmé cette prérogative, de même que Sixte IV. Sous ce pontife, la tutelle des Antiquités devient même un des rares secteurs de la vie citadine pour lequel l'Etat ne conteste pas les attributions des institutions municipales. Tout au long du XVIe siècle, l'administration capitoline paraît 
utiliser la rhétorique des Antiquités pour exalter son rôle dans l'administration de la ville ${ }^{5}$. Au $\mathrm{XVI}^{\mathrm{e}}$ siècle se développe aussi la tradition de collection des Antiquités parmi les grandes familles capitolines, Capodiferro ou Colonna par exemple. A une époque, ensuite, où la Papauté parvient à considérablement limiter les pouvoirs de la municipalité, on assiste à un repli vers le collezionismo, domaine privilégié de la construction d'une image de Rome chez les nobles capitolins. Pour le XVIIIe siècle, les Antiquités se confirment comme le lieu d'un repli municipal. Hanns Gross note ainsi : «La question des Antiquités était presque le seul argument susceptible de réveiller la paresseuse attention de la municipalité romaine $»^{6}$. Un groupe de collectionneurs, duquel émergent les figures d'Ennio Quirino Visconti et de Francesco Bianchini régit le sort des Antiquités romaines. Le $\mathrm{XIX}^{\mathrm{e}}$ siècle, entre archéologie désormais d'Etat et puissance des collectionneurs privés, souvent les princes de la noblesse romaine, hérite en grande part de cette évolution. Durant les dernières années du gouvernement pontifical, c'est Pietro Ercole Visconti qui est surintendant aux Antiquités et Beaux Arts. Mais avec les bouleversements de la fin de l'année 1870, le jeu est de nouveau ouvert entre l'Etat, désormais italien, et la municipalité, revigorée par l'application de la loi municipale italienne de 1865 , qui lui donne beaucoup de pouvoirs dont elle était privée sous l'administration pontificale. La municipalité romaine est donc renforcée dans ses prérogatives, et devient le refuge des intérêts pontificaux dans la ville perdue par Pie IX. Les rôles sont à la fois inversés et confirmés. On a toujours une volonté étatique de limiter les compétences municipales, mais c'est désormais dans l'action de la municipalité que se 
joue l'influence de la Papauté sur la ville. Alors que depuis des siècles l'action du gouvernement pontifical en matière d'Antiquités visait à limiter les prérogatives de la noblesse municipale, qui prétendait représenter le Popolo romano, il fallait désormais tout faire pour empêcher l'Etat de prendre la place laissée vacante et contester à la mairie la tutelle sur l'archéologie.

A partir de l'automne 1870, l'archéologie à Rome devient l'enjeu d'une lutte nouvelle, dans laquelle s'incarnent certains des principaux antagonismes animant la ville et l'histoire du pays. Entre l'Etat italien, qui supplante l'Etat pontifical, et la municipalité romaine, qui reprend sur le plan social et politique le flambeau du catholicisme dans la nouvelle capitale, s'ouvre un conflit destiné à durer de nombreuses années. L'archéologie en est un des enjeux. Entre image de la ville et de son devenir, contrôle des terrains, jeux rhétoriques de l'histoire et de l'appartenance, ou plus trivialement tutelle sur un secteur lucratif par bien des aspects, les restes de la Rome antique sont investis d'une nouvelle valeur.

Ce qui nous intéresse ici est de comprendre comment, entre 1870 et le début du XXe siècle, se joue autour de l'archéologie une rivalité spatiale pour la tutelle sur une portion considérable de l'espace de la ville. L'archéologie de Rome capitale est désormais bien connue dans sa dimension d'histoire de la discipline, voire d'histoire du paysage urbain. Le but de la présente démarche est plutôt de promouvoir une interprétation en termes d'histoire du territoire urbain et des institutions, ainsi que des groupes sociaux qui ont prise sur ce territoire. 
La Capitale de l'Etat italien, dans les premières années après 1870, est en effet à la fois dans une large mesure propriété foncière de l'aristocratie catholique, violemment hostile au gouvernement royal, et placée sous la responsabilité, du simple fait de l'application de la loi italienne de 1865 sur les Municipalités, de la Mairie de Rome, généralement contrôlée par ce même groupe social de l'aristocratie dite noire ${ }^{7}$. Pour l'Etat italien, il apparaît donc vital, dans une période où ne sont pas encore à l'œuvre les mécanismes de rapprochement qui marqueront les décennies suivantes, de prendre pied dans sa propre capitale. L'Etat a donc besoin de parvenir à contrôler l'espace de la ville capitale, et d'avoir prise sur les symboles utiles à la rhétorique d'une troisième Rome héritière de la Rome ancienne. L'idée de la Rome antique est omniprésente dans les luttes du Risorgimento qui s'achèvent avec la prise de la ville en 1870 et son accession au rang de Capitale du Royaume quelques semaines plus tard, et dès la fin de l'année 1870, un personnage comme Quintino Sella est avide de pouvoir exalter par la forme urbaine la filiation entre la Rome italienne et la Rome antique. Audelà de cette dimension cependant, c'est bien aussi de contrôle de l'espace urbain qu'il s'agit. Avec le chantier de régularisation du cours du Tibre, dont l'Etat, par l'intermédiaire du corps du Génie civil, prend rapidement le contrôle, au cœur de la ville, dès les premières semaines de Rome capitale, l'archéologie s'avère rapidement constituer, pour le gouvernement, le moyen d'acquérir une compétence d'ordre territorial dans les transformations du paysage urbain. Puisque la loi municipale italienne donne à la municipalité la responsabilité de la transformation de la ville, par les procédures de planification dites du Plan régulateur, l'Etat utilise 
rapidement l'archéologie comme argument destiné à délimiter une portion de territoire urbain au statut dérogatoire.

D'emblée, dès les dernières semaines de l'année 1870, l'archéologie est un des thèmes d'affrontement entre l'Etat et la mairie, désormais relais du Vatican et de la noblesse noire, qui réfute la tutelle italienne sur la ville pontificale. Il ne s'agit pas seulement de la question de la maîtrise de l'image reflétée dans l'Antique que l'on veut donner à la nouvelle capitale, mais aussi, au sens propre, d'une question de territoire.

Depuis la période napoléonienne au moins, l'idée que la zone archéologique centrale doit être préservée de la croissance urbaine et dégagée progressivement, est acceptée par à peu près tout le monde, et la Restauration pontificale n'est pas allée à l'encontre de cette tendance. En 1870, il n'est donc question pour personne d'urbaniser le forum et le Palatin, et même les pires spéculateurs dont la ville est riche à cette époque ne semblent pas l'imaginer. On n'a donc pas a priori de spéculation foncière pour expliquer la rivalité sur ces terrains. Ce qui l'explique, c'est que ni l'Etat ni la mairie ne veulent perdre la maîtrise d'une portion très significative du territoire urbain, autour de laquelle se jouent en revanche de nombreux enjeux de la croissance urbaine, voire de la spéculation. La zone archéologique centrale est à la fois une portion de ville, riche en symbole et bon support pour la rhétorique civique ou nationale, et la pièce centrale autour de laquelle s'articule l'urbanisation de la nouvelle capitale.

L'Etat, par l'intermédiaire de son représentant spécial, prend les devants dès les premiers jours. De même que le Génie civil prend en quelques semaines le contrôle du chantier du Tibre, à la suite de l'inondation de Noël, le 
Lieutenant général du Roi pour la place de Rome, Alfonso La Marmora, donne au Ministère de l'Instruction Publique la tutelle sur l'archéologie, donc les fouilles, et la portion du territoire urbain afférente. Sa décision de supprimer la Commissione pontificia per l'Antichità e le Belle Arti dès le 8 novembre $1870^{8}$, à un moment où la ville n'est pas encore tout à fait complètement sous le contrôle de l'armée italienne, est à replacer dans ce contexte. Cette décision est rendue publique le jour même de la prise de l'ancien Palais de Pie IX, le Quirinal, dont l'administration pontificale avait emporté les clefs. La conquête de Rome n'est pas encore tout à fait achevée, et certains secteurs de la rive droite sont encore l'objet d'une durable hésitation de la part de l'administration italienne. La commission pontificale serait en effet logiquement devenue municipale sans cette intervention, puisque ses membres avaient trouvé au Capitole un nouveau lieu d'expression, et qu'il leur aurait suffi de s'ériger en commission municipale pour poser de graves problèmes au gouvernement. Mais la mairie a été prise de vitesse et n'a pas eu le temps de déclarer la création d'une commission municipale. La commission pontificale est immédiatement remplacée par une institution ministérielle italienne. La création de la Sopraintendenz per gli scavi di Antiquità e per la custodia dei monumenti della provincia di Roma, dépendant du Ministère de l'Instruction publique, est donc le signe de la volonté précoce de l'Etat d'éviter une prolongation de la tutelle vaticane, via la mairie, sur la zone archéologique centrale. C'est Pietro Rosa qui est nommé à la tête de la nouvelle institution. Archéologue au service des Français au cours des décennies précédentes, il est pour Barbanera «le seul archéologue romain qui ne fût pas au service du Vatican » ${ }^{9}$. Il faut voir 
dans cette décision un changement qui va au-delà de l'appropriation étatique de la symbolique des Antiquités. La création à Rome de la surintendance est partie intégrante de la volonté du gouvernement italien de ne pas laisser à la municipalité le contrôle exclusif du territoire urbain. Pietro Rosa dirigeait depuis plusieurs années les fouilles du Palatin, pour le compte de Napoléon III, propriétaire, à titre personnel, des Orti Farnesiani. Le gouvernement italien le confirme dans ses fonctions sur ce chantier. Quintino Sella, Ministre des Finances de 1868 à 1873 et chantre de Rome capitale parmi la génération de la Droite historique au pouvoir, obtient que les terrains du Palatin soient rachetés par l'Etat.

Les archéologues catholiques et pontificaux sont évincés, et trouvent refuge, comme la majeure partie des intérêts catholiques, au Capitole. Pietro Ercole Visconti, son neveu Carlo Ludovico Visconti, De Rossi et Virginio Vespignani, qui étaient proches de Pie IX, investissent dans les activités municipales la passion qui jusque-là les animait. Ils restent ouvertement fidèles au pontife. Ugo Pesci, chroniqueur de ces années 1870 dans la nouvelle capitale, décrit avec sympathie ce mouvement de repli : pour lui, les archéologues «vaticani» ont bien raison de considérer avec dédain leurs collègues, pâles fonctionnaires, «statali », qui ont pris leur place ${ }^{10}$. Pesci ajoute que 1'Etat italien est loin de faire belle figure dans cette affaire. « La nouvelle Italie se trouvait en position de faiblesse, du point de vue de l'archéologie officielle, face à la science archéologique vaticane $»^{11}$. Seul Rosa trouve éventuellement grâce à ses yeux.

L'idée de la création d'une commission archéologique municipale naît de ce repli vers la mairie. Mais, alors que, pour d'autres domaines, Etat et mairie 
rivalisent de vitesse et de ruse dans la création de commissions concurrentes, pour l'archéologie, le gouvernement parvient facilement à évincer la mairie. Dans le champ des Antiquités, la mairie n'aura jamais cette capacité de contester vraiment l'emprise étatique. L'archéologie est ainsi le théâtre de la première victoire du gouvernement italien dans l'espace urbain, après la victoire militaire du $X X$ Settembre. Face à la nécessité de ne rien faire qui puisse donner à Pie IX un prétexte pour quitter la ville, les gouvernements italiens de Minghetti, puis de Lanza, jouent sur du velours. Il n'est pas question d'avancer d'une manière brusque. De même que les soldats hésitent plusieurs jours avant de passer les ponts du Tibre, que les policiers du Ministre de l'Intérieur restent discrets pendant plusieurs mois, la prise de contrôle de l'espace urbain ne peut en aucun cas se faire par l'éviction trop brutale d'une mairie dont la loi de 1865 affermit les prérogatives. L'archéologie est à ce moment un moyen précieux de gagner la tutelle sur une portion importante de l'espace de la ville.

La mairie tarde à réagir, et le conflit semble mois âpre que ne le laisse entendre Barbanera ${ }^{12}$. Malgré le manque de continuité dans la politique ministérielle, marquée par une succession de ministres de l'Instruction publique dans la première moitié des années 1870, l'Etat ne voit jamais remise en question sa domination sur le secteur. Contrairement au domaine des travaux publics, le Capitole ne dispose guère, en la matière, du personnel adéquat, ni d'une tradition bureaucratique susceptible de permettre de rapides réponses. Les archéologues municipaux sont isolés et n'ont rien de fonctionnaires. Alors que pour le Tibre la mairie cause d'immenses soucis au gouvernement, et joue de tous ses arguments pour 
repousser la volonté de tutelle étatique, pour le domaine de l'archéologie, la réaction est lente et inappropriée. On proteste, mais on ne trouve pas la rhétorique administrative adaptée. La Commissione archeologica municipale n'est créée que le 24 avril $1872^{13}$, un an et demi après la prise de contrôle par l'Etat du secteur de l'archéologie romaine. Elle est certes composée des archéologues catholiques évincés un an et demi plus tôt, De Rossi, Visconti, Vespignani, auxquels on adjoint Rosa, Lanciani, disciple de Visconti, Castellani et Vitelleschi, mais ne parvient pas à affirmer sa tutelle sur la portion de territoire urbain concernée au premier chef par l'archéologie: la zone archéologique centrale. En fait, la Commission municipale ne bénéficie ni de l'énergie d'un ingénieur comme Angelo Vescovali, qui s'occupe du Tibre, ni de celle de nobles collectionneurs qui n'ont pas encore compris que c'est sur le terrain administratif et juridique que se joue la rivalité. Les archives de cette commission, à l'Archivio Storico Capitolino, sont d'ailleurs extrêmement décevantes : pour les trois premières années d'activité, on n'a guère trace que de réunions dans lesquelles on discute des trouvailles sur les chantiers (de voirie) municipaux, hors de la zone placée sous la tutelle de l'Etat. Même pour les zones municipales, aucune vision de la ville ne ressort des travaux de la Commission. Sa seule tâche est l'accompagnement des chantiers de construction et la collecte des objets trouvés à l'occasion de la mise en place des nouveaux quartiers. Quant au rapport de la Commission au Conseil municipal, présenté en 1875, il ne fait que 6 pages pour les années 18721873-1874 ! Le budget de 50000 lires qui lui avait été attribué a été dépensé dans le financement d'un timide repli vers les terrains municipaux ${ }^{14}$. Au 
cours des années 1870 et 1880, la Commission archéologique municipale se contente de réguler le flux des découvertes archéologiques ayant lieu dans les quartiers en construction, Esquilin essentiellement ${ }^{15}$. Il s'agit d'augmenter les collections municipales, et en aucun cas de contribuer au façonnement de l'espace de la ville. La Commission archéologique municipale a abandonné toute prétention sur les terrains de l'archéologie d'Etat. Cette attitude perdure pendant de longues années. Seules, peut-être, les protestations contre la décision du Président du Conseil Depretis, en 1883, de construire le monument à Victor-Emmanuel au dos de la colline du Capitole, parviennent à donner à la rhétorique de la commission archéologique municipale un certain écho. L'archéologie sert alors pour une saison d'argument à la mairie pour protester contre l'écrasant monument ${ }^{16}$. En dehors de cet épisode, la routine domine. Le bulletin que publie la commission municipale se contente de présenter les objets trouvés dans les chantiers de construction des divers quartiers de l'Esquilin. Les vols sont évidemment nombreux, que n'explique pas entièrement l'argument selon lequel «il est difficile de surveiller 10000 ouvriers $»^{17}$. Ce qui est trouvé dans des chantiers de propriété privée est catalogué avant d'être livré au marché international ${ }^{18}$. Les objets retrouvés en terrain municipal servent à la constitution du Musée municipal du Palais des Conservateurs au Capitole.

Quant au Ministère de l'Instruction publique et aux services de l'archéologie du Sénateur Fiorelli et du Professeur Barnabei, ils ont l'habileté d'appeler à siéger dans la commission ministérielle les principaux archéologues catholiques et municipaux, où ils formeront une minorité inoffensive. Les deux Visconti, De Rossi et Vespignani connaissent de la 
sorte un parcours encadré, et leur talent d'archéologue n'est guère mis au service de la mairie.

En 1874, Rosa est écarté, à la suite des cafouillages du chantier du Colisée, durablement inondé par la rupture de plusieurs canalisations. Les archives des services techniques municipaux prouvent que l'on prit l'affaire avec ironie au Capitole, et il est certain que les ingénieurs des services techniques municipaux, plus prestes que les archéologues municipaux dans la contestation de la tutelle étatique sur la ville, n'ont rien fait pour aider De Rosa à comprendre d'où venaient les égouts qui tout à coup se déversaient dans le Colisée ${ }^{19}$. A partir de 1876, avec l'arrivée de la Gauche au gouvernement, le conflit avec la mairie reprend brièvement, au sujet surtout des églises. Le Ministre Michele Coppino suscite une profonde irritation au Capitole à ce sujet, mais le destin de la zone archéologique centrale n'est pas vraiment en jeu.

Il l'est en revanche dans la question du plan régulateur, qui anime la vie municipale tout au long de la décennie ${ }^{20}$. Dans la pratique municipale italienne, le dessin d'un Plan régulateur est en effet l'acte essentiel dans la manifestation de la volonté de décider des grandes orientations de l'évolution de la ville. Entre 1870 et la Première Guerre mondiale, Rome connaît trois plans régulateurs : 1873, 1883, 1909. Il est donc légitime de se demander quelle est la place accordée à l'archéologie, et particulièrement à la zone archéologique centrale dans ces documents. On peut aussi se demander dans quelle mesure la commission municipale d'archéologie parvient à se faire entendre du bureau de la planification. 
Mais dans le contexte municipal romain, surtout pour les années 1870-1890, ces questions trouvent rapidement une réponse claire : il n'y a pas de coordination, la zone archéologique est largement ignorée par le plan. Le plan lui-même, d'ailleurs, ne devient vite que l'instrument fragile d'éphémères pouvoirs municipaux progressistes (en 1873 et en 1883), vidé de sa substance dès que la noblesse catholique conservatrice et lotisseuse retrouve ses prérogatives au Capitole. Les Plans régulateurs de Rome capitale laissent donc la zone archéologique dans le flou.

Une des explications à la difficulté fondatrice pour la commission archéologique à trouver un positionnement dans l'appareil municipal est à chercher, au-delà des conflits de personnes, dans la maturation des débats sur la rédaction du Plan Régulateur. La commission archéologique est composée de personnages hostiles aux planificateurs municipaux progressistes de 1873 et 1883 , et ne travaille qu'en collaboration avec le bureau dit des nouveaux quartiers, qui planifie de fait la ville en construction sur l'Esquilin hors de la procédure légale de planification. Cette attitude est politiquement logique, mais au fond en vient à desservir la mairie, qui laisse le champ libre à l'archéologie d'Etat. On peut même dire qu'à partir de 1887, l'Etat planifie la ville à partir de la zone archéologique qu'il contrôle.

Etant le document unique de l'action municipale sur le territoire urbain, le Plan Régulateur aurait dû comprendre d'emblée une feuille consacrée à l'archéologie. Cela aurait été le moyen pour la mairie d'affirmer son pouvoir sur la ville et le devenir de la zone archéologique, quitte à se heurter à la volonté de l'Etat. Or, si plusieurs projets de plan déposés par des privés 
comprennent la zone archéologique, ceux rédigés par les services techniques de la mairie l'ignorent. La zone archéologique, et sa vocation à être préservée de la spéculation, sont décrites dans le texte, mais cette zone ne fait pas l'objet d'une catégorisation sur le dessin du plan.

On lit généralement l'histoire de l'archéologie dans son rapport à la planification urbaine de Rome capitale comme l'histoire des destructions qui ont marqué la construction du quartier de l'Esquilin ${ }^{21}$. Mais cette histoire peut aussi être lue en creux : la zone archéologique comme lieu où le plan n'a pas prise, et comme espace premier d'une véritable planification urbaine gouvernementale, avec la volonté de créer de la ville, avec ses jardins, ses avenues arborées et ses modes d'articulation aux différents quartiers.

La municipalité ignore tellement cet espace qu'il est prévu dans le Plan approuvé en 1873 que la zone serait survolée par un pont. La zone archéologique apparaît à ce moment comme espace perdu pour la mairie, à l'image du Tibre, que l'on ne peut qu'enjamber. Et encore cette démarche n'est-elle que l'idée, vite abandonnée, des progressistes de l'administration Pianciani. L'enjeu unique pour la mairie aux abords de la zone archéologique devient rapidement l'urbanisation de la via Cavour, qui relie le quartier de l'Esquilin et de la gare des Thermes (Termini) au Colisée.

Pour les archéologues municipaux, la difficulté à se faire entendre des ingénieurs de la planification, Viviani en tête, explique peut-être la création d'une commission séparée en 1872. Mais l'indifférence et le manque de vision administrative et politique est sans doute l'explication principale. Les archéologues municipaux ne s'intègrent pas dans l'appareil bureaucratique 
capitolin, et ne se mettent pas au service du combat que mène celui-ci pour préserver son emprise sur la ville. La commission archéologique arrive trop tard, et en se plaçant hors des procédures de la planification, seul espoir municipal de prise sur le territoire urbain, elle limite grandement ses capacités d'action.

En 1872 déjà, un architecte et pamphlétaire romain, Giambattista Demora, avait souligné ce risque pour la mairie de ne pas maîtriser l'évolution d'une portion importante du territoire urbain ${ }^{22}$. Dans un opuscule envoyé au conseiller municipal progressiste Pianciani, qui devient maire de novembre 1872 à juillet 1874, Demora se plaint de l'incapacité des planificateurs municipaux à tenir compte des enjeux de l'archéologie, et note déjà que c'est par le Piano Regolatore que la mairie peut reprendre la main en la matière. Demora a compris que la loi municipale italienne donne à la municipalité romaine ce formidable instrument qu'est la procédure de planification, et qu'omettre d'y inclure la ville dans son ensemble revient d'emblée à dessaisir la mairie d'une part de ses prérogatives sur le territoire urbain. L'appel a peu d'écho. Les antagonismes internes au milieu capitolin l'expliquent largement.

Face donc à une commission municipale faible, à une zone archéologique abandonnée par la planification municipale, les visées étatiques des ministres Correnti puis sous la gauche Coppino ont donc eu la voie libre. Les années 1870 sont donc marquées par l'affermissement du contrôle étatique sur la zone archéologique centrale et lors des discussions sur les projets de plan régulateur au début des années 1880, Demora republie son opuscule, et décrit la situation nouvelle en deux mots: "Oh delusione"23. 
Le Piano Regolatore de 1883, publié après l'échec de la seconde parenthèse Pianciani et lors du retour de la majorité capitoline proche du Vatican, avec Torlonia, ne prévoit plus de pont, mais n'est pas plus clair au sujet d'une zone archéologique que la mairie semble avoir abandonnée à l'Etat. Torlonia, prince, banquier, spéculateur et hôte assidu des salons du Vatican, se consacre prioritairement à l'extension des quartiers nouveaux à l'Esquilin, ainsi qu'à l'ouverture aux lotisseurs des horizons du quartier des Prati di Castello.

Demora, passionné de ces questions, l'a compris, et ne manque pas de faire un éloge appuyé du ministre de l'instruction publique Guido Baccelli. C'est bel et bien du côté ministériel que reposent les espoirs d'une planification de la zone archéologique en tant qu'espace urbain. Dans la seconde moitié des années 1880, Lanciani, secrétaire de la commission municipale, fouille le forum, mais les jeux sont faits en matière de tutelle institutionnelle sur le territoire urbain, d'autant plus que l'action de l'Etat s'est renforcée par la création de la Commissione Reale, en 1881, présidée par le sénateur napolitain Fiorelli, déjà responsable de l'archéologie au Ministère depuis 1875, dont le rôle était sans doute d'effacer un peu plus l'influence de Rosa. Cette commission rend en 1887 un Piano per la sistemazione della zona monumentale riservata di Roma. Les jeux sont faits, et l'évolution vers la passegiata archeologica, terminologie qui s'impose alors, chère à Baccelli, est en marche. La zone archéologique d'Etat est à partir de ce moment considérée comme un parc urbain.

De 1870 à 1890 l'évolution se fait donc par dérogation au statut des terrains urbains, dont la destination devrait être sanctionnée par le plan régulateur. 
Pour la fin des années 1880, la césure entre mairie et Etat est moins tranchée. Le rapport de l'Etat au monde catholique l'est moins également. C'est de son poste d'adjoint au maire chargé de l'instruction que l'ex et futur ministre de l'Instruction publique Baccelli relance en 1887 son idée de jardin public archéologique.

Lors de la séance du 17 janvier 1887 du Conseil municipal, il fait voter le texte suivant : «Le Conseil communal de Rome, déclarant utile et élégant pour la Capitale du Royaume le projet de relier les monuments antiques de la zone méridionale de la ville par des jardins publics et de grandes avenues arborées, demande à ce que le gouvernement royal promeuve les mesures légales nécessaires et mette des fonds à disposition de cette œuvre, dans la juste mesure de ses moyens économiques $»^{24}$. La zone archéologique romaine, chez Baccelli, est traitée à la fois comme parc urbain et comme promenade culturelle ${ }^{25}$. A la Chambre des Députés, le 14 juillet 1887, Baccelli et Ruggiero Bonghi font voter la première loi sur la zone $\operatorname{archéologique}^{26}$. Celle-ci comprend le Forum, le Palatin, le Colisée, les Thermes de Trajan, le temple de Claude, et la via Appia jusqu'à la Porte San Sebastiano. La mise en œuvre du programme est prévue sur 10 ans et une part importante de l'espace de la ville est concernée. Les expropriations en vue de la réalisation de ce parc, menées grâce à la loi Baccelli-Bonghi de 1887, semblent se faire dans un climat plus consensuel entre la mairie et le ministère $^{27}$. Une commission, présidée par Fiorelli, fait la liste des propriétés à exproprier. La commission est composée de membres nommés par l'Etat et la mairie: Fiorelli, Directeur Général des Antiquités au Ministère de l'Instruction publique; Guido Baccelli ; Bonghi, 
Bongiovannini, Canevari, Bernabei (Directeur des Musées Nationaux) pour l'Etat ; Mario Ceselli, adjoint au maire ; De Rossi, Lanciani, Vitelleschi et Camillo Re pour la municipalité.

A l'échéance du terme de dix ans, le ministre de l'Instruction publique Gianturco fait voter une loi de prorogation des dispositions de la loi de 1887, au prix cependant d'une réduction considérable du périmètre concerné. La nouvelle zone, loin d'arriver aux murs de la ville, ne dépasse pas les termes de Caracalla.

En 1898, Baccelli est cependant de nouveau Ministre de l'Instruction publique, et peut relancer le projet, par la loi du 18 décembre. Un budget de 1,8 millions de lires est attribué. A la même époque Giacomo Boni est nommé à la tête de l'archéologie gouvernementale à Rome. A un moment de la carrière du grand archéologue où il comptait se rendre à Constantinople, il est rappelé sur le Forum pour une troisième grande campagne gouvernementale, après celles de Rosa puis de Lanciani. Dans un contexte où l'opposition avec la mairie a quasiment disparu de ce terrain, Boni installe une commission composée de l'architecte Sacconi, et de Lanciani, Gatti et Hülsen, afin de décider de l'orientation des travaux. De concert avec Baccelli, il parvient à relancer dans le public l'idée de la promenade archéologique, grâce à la complicité du journal La Tribuna, qui, opportunément, suscite le débat et réclame une remise à jour du grand dessein $^{28}$. Le 18 décembre 1898, Guido Baccelli, ministre de l'Instruction publique, dont le passage dans les rangs des Conseillers municipaux capitolins n'a pas peu fait pour rapprocher les deux traditions archéologiques, obtient un financement de 1,8 millions de lires, apporté 
pour moitié par l'Etat et pour l'autre moitié par la mairie. C'est le véritable démarrage des travaux paysagers à grande échelle. En un an, le nombre d'ouvriers passe de 20 à plus de 100 . Baccelli parvient à passionner la Nation, organise des visites du chantier pour les Députés ${ }^{29}$. Boni rend compte au Ministre de l'avancement des travaux au jour le jour. Les difficultés avec la mairie sont définitivement aplanies, puisqu'il n'y a plus de véritable enjeu, et Boni parvient même à faire déplacer les rails du tramway dans des délais raisonnables. 1900 est le moment le plus fastueux de cette période, avec la visite du Roi. Au mois de juin cependant, le départ de Baccelli du Ministère ralentit les travaux, et les archéologues peinent à convaincre son successeur Gallo du bien fondé de la tâche. C'est le soutien du roi, et l'argument du chômage infligé aux ouvriers, qui relance Boni. L'archéologue quitte cependant Rome en 1902 pour sa ville de Venise, où s'est effondré le campanile de Saint-Marc. Mais on peut dire qu'au tournant du siècle la zone archéologique centrale a pris place dans l'espace urbain et s'impose comme domaine gouvernemental au cœur de la capitale. Le domaine privilégié de l'action étatique au cœur de la capitale n'est plus guère contesté. Lorsque l'archéologie est le prétexte d'une rivalité entre institutions, c'est pour d'autres aires du territoire urbain. La mairie conteste ainsi le sort réservé aux Antiquités du Tibre, et tente par ce biais de trouver de nouveau le moyen de donner son avis sur la question du fleuve. Mais entre le Forum et les thermes de Caracalla, les archéologues de l'Etat travaillent en paix.

L'essentiel des expropriations a lieu sous le ministère Baccelli de la fin du siècle, dans le gouvernement Pelloux en 1898, ainsi que vers 1907, quand la 
loi du 11 juillet élargit de nouveau le périmètre de la zone archéologique, pour revenir presque à la définition de 1887. Six millions de lires sont prévus pour les expropriations (4 pour l'Etat, 2 pour la mairie). Il est à noter toutefois que, dans ce processus d'expropriation, la commune ne maîtrise vraiment aucun des stades de la décision, bien qu'elle participe au financement à hauteur d'un tiers. Le parc archéologique, en ce sens, présente une configuration similaire à celle du chantier du Tibre: la municipalité est exclue des processus de décision, mais nullement dispensée de contribuer à la dépense.

En 1907 est créée une nouvelle Commissione Reale, présidée de nouveau par Guido Baccelli, dans laquelle siège le Professeur Rodolfo Lanciani, désormais Sénateur. Il s'agit à partir de ce moment de préparer le paysage urbain de la capitale pour les festivités de $1911^{30}$. L'archéologie, et le projet de promenade archéologique, sont alors clairement mis au service de l'image de Rome capitale que veut donner le gouvernement italien. Sur «ses terres» (le périmètre de l'exposition, le monument à VictorEmmanuel, la zone archéologique centrale), l'Etat engage un important programme de mise en scène de sa propre tutelle sur la ville, qui confirme la place de la zone archéologique comme territoire étatique au cœur de la ville municipale. L'endroit est le lieu d'expression des idées ministérielles. Baccelli dirige le mouvement jusqu'en 1910. L'essentiel des travaux consiste à surélever le sol du forum, et à le mettre à l'abri des inondations. En ce sens, l'Etat se contredit lui-même dans ses deux bastions, le chantier du Tibre mettant en danger celui de l'archéologie. Mais en 1910 ces conflits n'ont plus lieu d'être. La noblesse capitoline et le monde catholique en 
général ont été largement intégrés à la vie de la nation, et la rivalité entre la mairie et l'Etat s'est tout à fait banalisée, ne recouvrant plus les enjeux des décennies précédentes.

Avec les lois de 1914 et de 1917, le périmètre de la zone archéologique est encore consolidé, les Forums impériaux et le Capitole y étant intégrés ${ }^{31}$. En 1914, la Commissione reale (Baccelli, Rocco, Lanciani, Mercadante, Vignali) produit une belle carte de la zona monumentale di Roma, et invite la mairie à prendre livraison de la voirie. Après plusieurs incidents avec la municipalité sur les conditions de cette mise à disposition, et sur le statut des voies (conflit désormais inclus dans la normalité des relations entre administrations centrale et locale), la zone est pleinement intégrée à la ville en $1917^{32}$.

Sous le gouvernement fasciste, les idées de Baccelli sont à la fois consolidées et déviées : la zone archéologique centrale est plus que jamais protégée de l'urbanisation (et même étendue aux dépens de la ville), mais en même temps soumise à une mise en scène urbaine accrue. Mais plus que la scénographie fasciste, jouant de la rhétorique de l'Antiquité, c'est l'idée de circulation au travers de la zone qui en sape les fondements. La transformation des voies de promenade imaginées par Baccelli en voies de communication entre les deux parties de la ville pervertit considérablement l'idéal de départ et marque considérablement, et durablement, le paysage urbain de la capitale ${ }^{33}$. La zone archéologique centrale est de moins en moins un parc, et les larges avenues créées, ainsi que les viali de Baccelli détournés de leur vocation récréative, contribuent à l'ouverture de la ville au trafic. La ville moderne, dans son rapport à la ville antique, y perd un espace 
dont Baccelli avait mis en scène à la fois la quiétude et l'intégration à l'espace urbain ${ }^{34}$.

${ }^{1}$ Sur cet épisode : R. T. Ridley, The Pope's Archeologist : the life and times of Carlo Fea, Rome, 2000, p.221. Consalvi, en 1818, avait souhaité transférer la compétence en matière de tutelle des Antiquités au Trésorier.

${ }^{2}$ Edit du 7 avril 1820. Le texte comprend 61 clauses. Voir Ridley, op. cit., p.221.

${ }^{3}$ Edit du 27 Septembre 1827. Voir Ridley, Op. cit., p.309

${ }^{4}$ A ce sujet : M. Franceschini, La magistratura capitolina e la tutela delle Antiquità di Roma nel XVI secolo, dans Archivio della Società Romana di Storia Patria, 1986, p.141-150

${ }^{5}$ Voir Franceschini, loc. cit.

${ }^{6}$ H. Gross, Roma nel Settecento, Rome-Bari, 1990, p.361.

${ }^{7}$ Sur le contexte politique des premières années de Rome capitale: A. Caracciolo, Roma capitale. Dal Risorgimento alla crisi dello Stato liberale, Rome, 1956.

${ }^{8}$ Sur ce point, voir: M. Barbanera, L'archeologia degli italiani. Storia, metodi e orientamenti dell'archeologia classica in Italia, Rome, 1998.

${ }^{9}$ Voir : Barbanera, op. cit., p.36.

${ }^{10}$ U. Pesci, I primi anni di Roma capitale. 1870-1878, Florence, 1907, p.378 et suivantes.

${ }^{11}$ Idem, p.378

${ }^{12}$ Barbanera, op. cit., p.37.

${ }^{13}$ Archivio Storico Capitolino, Atti, 1872. 
${ }^{14}$ Archivio Storico Capitolino, 11646 (44), Ai signori consiglieri comunali.

Memoria della Commissione archeologica municipale, 28 Gennajo 1875.

15 Voir: A.M. Ramieri, Enrico Stevenson: cenni biografici ed inediti documenti d'archivio della Commissione archeologica comunale, dans Rivista di Archeologia Cristiana, 1998, 2, p.329-351. Stevenson était un des principaux disciples de De Rossi.

16 Archivio Storico Capitolino, Atti Consiglio, Avril-Mai 1883 et Commissione Archeologica, 17 avril 1883.

${ }^{17}$ Idem.

${ }^{18}$ Idem.

${ }^{19}$ Archivio Storico Capitolino, Ufficio V, Direzione, Tit. XV, 1874.

${ }^{20}$ Sur les plans régulateurs de Rome capitale : I. Insolera, Roma moderna. Un secolo di storia urbanistica. 1870-1970, Turin, 1993.

21 Voir: L. Quilici, La tutela archeologica nei Piani regolatori e nella legislazione, dans G. Pisani Sartorio (éd.), L'archeologia in Roma capitale tra sterro e scavo, Venise, 1983, p.48-74.

22 G. Demora, Il Piano Regolatore di Roma e le antichità classiche. Osservazioni e proposte, Rome, 1872.

${ }^{23}$ Idem, 1882.

${ }^{24}$ Archivio Storico Capitolino, Atti, Seduta 17-1-1887.

Voir aussi : G. Lugli, La zona archeologica di Roma, Rome, 1924.

${ }^{25}$ Sur les parcs urbains, voir: G. Pettena, Olmsted. L'origine del parco urbano e del parco naturale contemporaneo, Florence, 1996.

${ }^{26}$ Loi n ${ }^{\circ} 430 \mathrm{du}$ 14-7-1887. 
${ }^{27}$ Voir : Camera dei Deputati, Atti, Leg. XVI, Sess. Ia, débat du 5 Juillet 1887.

${ }^{28}$ Voir : E. Tea, Giacomo Boni nella vita del suo tempo, Milan, 1932, t.II, p.9.

${ }^{29}$ Comme en janvier 1899. Tea, op. cit., p.19.

${ }^{30}$ Voir : G. Piantoni, Roma 1911, Rome, 1980.

${ }^{31}$ Lois n$^{\circ} 1111$ du 19/7/1914 et $n^{\circ} 1715$ du 12/10/1917.

${ }^{32}$ En 1914, en effet, Fausto Aphel, Commissaire extraordinaire, qui fait office de maire de la ville dans une situation de crise politique au Capitole, refuse la mise à disposition de la voirie de la zone archéologique. L'argument principal en est la mauvaise couture avec la via Cavour, près du Colisée. Sur cet épisode : M. De Vico Fallani, Storia dei giardini pubblici di Roma nell'Ottocento, Rome, 1992, p.214.

33 Sur la période fasciste: A. Cederna, Mussolini urbanista. Lo sventramento di Roma negli anni del consenso, Rome-Bari,, 1981 et I. Insolera et F. Perego, Storia moderna dei Fori di Roma, Rome-Bari, 1999. Sur les destructions : L. Barroero, A. Conti, A. Racheli et M. Serio, Via dei Fori imperiali, Venise, 1983. Sur les tentatives ultérieures de recoudre le paysage urbain autour de la zone archéologique: L. Benevolo et F. Scoppola, Roma : l'area archeologica centrale e la città moderna, Rome, 1988. Voir aussi : L. Benevolo, Studio per la sistemazione dell'area archeologica centrale, Rome, 1985. Ainsi que: R. Panella (dir.), Roma : città e Foro. Questioni di progettazione del centro archeologico monumentale della capitale, Rome, 1989 et A.M. Seronde-Babonaux, La Rome contemporaine dans ses rapports avec la ville antique, dans $\mathrm{R}$. 
Chevalier (dir.), Présence de l'architecture et de l'urbanisme romains. Hommage à Paul Dufournet, Paris, 1983, 419-427 et A. Clementi (dir.), Il senso delle memorie in architettura e urbanistica, Rome-Bari, 1990.

${ }^{34}$ Pour le cas de Syracuse : G. Voza, La città antica e la città moderna, dans S. Adorno (dir.), Siracusa. Identità e Storia. 1861-1915, Palerme, 1998, p.249-260.

Denis Bocquet, Ecole française de Rome 